\title{
Half Cuts em Grafos Bipartidos Completos
}

\author{
Rubens A. Sucupira ${ }^{1}$, Sulamita Klein ${ }^{2}$, Luerbio Faria ${ }^{1}$ \\ ${ }^{1}$ Instituto de Matemática e Estatística - Universidade do Estado do Rio de Janeiro (UERJ)
}

${ }^{2}$ COPPE Sistemas - Universidade Federal do Rio de Janeiro (UFRJ)

\begin{abstract}
A graph is Half Cut if it admits an edge cut with exactly $\left\lceil\frac{m}{2}\right\rceil$ edges. It is known that graceful graphs are Half Cut and that complete bipartite graphs are graceful. In this paper we give an alternative proof that complete bipartite graphs are Half Cut, exhibiting an edge cut with exactly $\left\lceil\frac{m}{2}\right\rceil$ edges.
\end{abstract}

Resumo. Um grafo é Half Cut se admite um corte de arestas de cardinalidade $\left\lceil\frac{m}{2}\right\rceil$. É sabido que grafos graciosos são Half Cut e os grafos bipartidos completos são graciosos. Neste artigo damos uma prova alternativa de que os grafos bipartidos completos são Half Cut, exibindo um corte de arestas de cardinalidade $\left\lceil\frac{m}{2}\right\rceil$.

\section{Introdução}

Dados um grafo $G=(V, E)$ com $n$ vértices e $m$ arestas, e um subconjunto não vazio próprio $S \subset V$, definimos o corte de arestas $\partial S$ como o conjunto formado por todas as arestas com exatamente um extremo em $S$. Dizemos que um corte de arestas $\partial S$ tal que $|\partial S|=\left\lceil\frac{m}{2}\right\rceil$ é um half cut. Um grafo $G=(V, E)$ é do tipo Half Cut se admite um half cut. Um modo de caracterizar um corte de arestas é através da partição associada a esse corte $V=S \cup(V \backslash S)$ que também representamos por $V=(S, V \backslash S)$.

Como exemplo de grafo Half Cut podemos considerar o grafo ciclo $C_{4}$ no qual encontramos cortes de arestas com cardinalidade $2=\left\lceil\frac{4}{2}\right\rceil$. Em [Sucupira et al. 2017], exibimos partições Half Cut para quaisquer caminhos $P_{n}$ e mostramos que só existem tais partições para os ciclos $C_{n} \operatorname{com} n \equiv 0(\bmod 4)$ ou $n \equiv 3(\bmod 4)$. Um grafo $G=(V, E)$ é bipartido completo se seu conjunto de vértices $V$ pode ser particionado em dois conjuntos independentes $V_{1}$ e $V_{2}$, de modo que cada vértice de $V_{1}$ é adjacente a todo vértice de $V_{2}$. Se $\left|V_{1}\right|=p$ e $\left|V_{2}\right|=q$, representamos o grafo bipartido completo correspondente por $K_{p, q}$. No contexto do artigo atual, o grafo $C_{4}$ pode ser visto como $K_{2,2}$ e apresenta uma outra maneira de construção da partição Half Cut. A Figura 1 a seguir apresenta essas duas partições.

Outros exemplos de grafos Half Cut são as rodas $W_{n}$ que têm $m=2 n$ arestas e podem ser decompostas em uma estrela central $S_{n}$ e um ciclo $C_{n}$, de modo que a partição Half Cut fica trivialmente determinada pela decomposição $V=(\{c\}, V \backslash\{c\})$ na qual $c$ é o centro da estrela $S_{n}$.

Neste artigo mostramos que todo grafo bipartido completo $K_{p, q}$ é do tipo Half Cut, exibindo explicitamente um corte de arestas de cardinalidade $\left\lceil\frac{m}{2}\right\rceil$. 


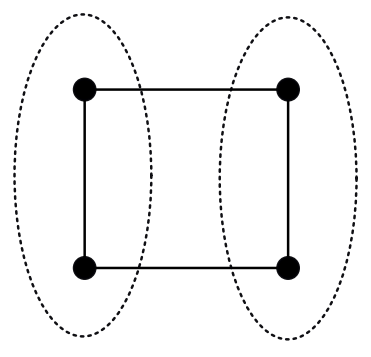

(a)

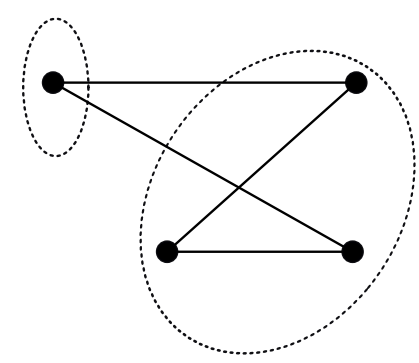

(b)

Figura 1. (a) Half cut de $C_{4}$ e (b) Half cut de $K_{2,2}$.

\section{Grafos Graciosos}

Uma rotulação graciosa de um grafo $G=(V, E)$ é composta por uma rotulação dos vértices $f: V \rightarrow\{0,1, \cdots, m\}$ e uma rotulação das arestas $f_{\gamma}: E \rightarrow\{1,2, \cdots, m\}$ definida por $f_{\gamma}(\{u, v\})=|f(u)-f(v)|$ ambas injetivas. Se $G$ admite uma rotulação graciosa, então $G$ é dito um grafo gracioso. A Figura 2 apresenta as rotulações graciosas de $C_{4}$ e de $K_{2,3}$.

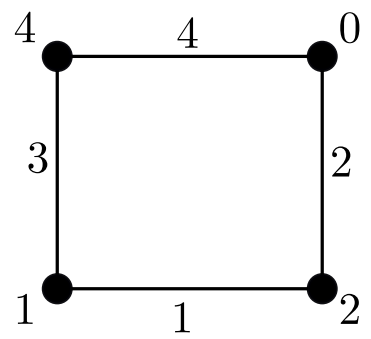

(a)

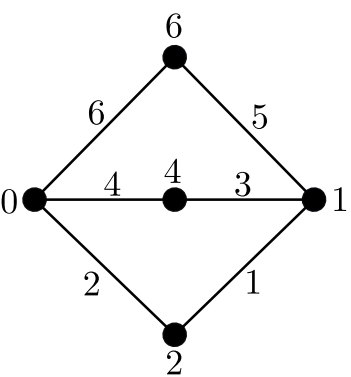

(b)

Figura 2. Rotulações graciosas de (a) $C_{4}$ e de (b) $K_{2,3}$.

[Golomb 1972] provou que todo grafo gracioso é do tipo Half Cut. A demonstração de Golomb pode ser encontrada em [Sucupira et al. 2017]. [Rosa 1966] e [Golomb 1972] provaram que todo grafo bipartido completo é gracioso, ou seja, já era conhecido o fato de que os grafos bipartidos completos são Half Cut.

\section{Grafos Bipartidos Completos}

A seguir demonstramos que os grafos bipartidos completos são Half Cut fornecendo um processo que define explicitamente uma partição Half Cut para tais grafos.

Teorema 3.1. Todo grafo bipartido completo é do tipo Half Cut.

Demonstração. Seja $K_{p, q}$ um grafo bipartido completo. Temos dois casos a considerar: No primeiro caso, pelo menos uma das cardinalidades $p$ ou $q$ é par; e no segundo caso, ambas as cardinalidades $p$ e $q$ são ímpares.

Primeiro caso: Sem perda de generalidade, consideremos $p$ par, já que o caso $q$ par se trata de maneira análoga. Então $p=2 k$ para algum inteiro positivo $k$. Vamos 
considerar $V_{1}=\left\{u_{1}, u_{2}, \cdots, u_{k}, u_{k+1}, \cdots, u_{p}\right\}$. Como todos os vértices de $V_{1}$ são adjacentes a todos os de $V_{2}$, todos os vértices $u_{i} \in V_{1}$ têm o mesmo grau $q$, ou seja, $K_{p, q}$ tem exatamente $p q$ arestas, mais especificamente, $m=2 k q$ e assim $\left\lceil\frac{m}{2}\right\rceil=k q$. Uma maneira simples de obter um corte de arestas com tal cardinalidade é formar a partição $V=(A, B) \operatorname{com} A=\left\{u_{1}, u_{2}, \cdots, u_{k}\right\}$ e $B=V \backslash A$, ou seja, transferir metade dos vértices de $V_{1}$ para a outra parte da partição, eliminando metade das arestas de corte. A Figura 3 abaixo ilustra essa situação com exemplos de partições Half Cut para os grafos bipartidos completos $K_{4,3}$ e $K_{6,4}$.

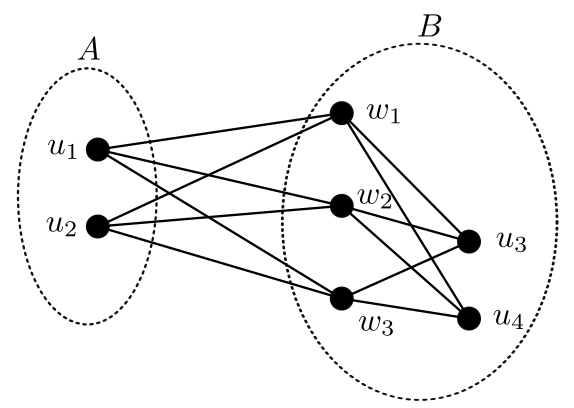

(a)

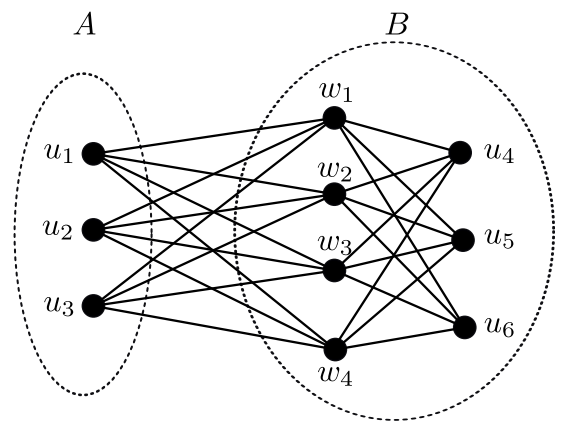

(b)

Figura 3. (a) Half cut de $K_{4,3}$ e (b) Half cut de $K_{6,4}$.

Segundo caso: Se $p$ e $q$ são ambos ímpares, existem inteiros positivos $i$ e $j$ tais que $p=2 i+1$ e $q=2 j+1$. Desse modo, o grafo bipartido completo $K_{p, q}$ tem $m=$ $(2 i+1)(2 j+1)$ arestas e $\left\lceil\frac{m}{2}\right\rceil=2 i j+i+j+1$. Nesse caso consideremos $V_{1}=$ $\left\{u_{1}, u_{2}, \cdots, u_{i+1}, \cdots, u_{p}\right\}$ e $V_{2}=\left\{w_{1}, w_{2}, \cdots, w_{j+1}, \cdots, w_{q}\right\}$ e a partição $V=(A, B)$ na qual $A=\left\{w_{1}, w_{2}, \cdots, w_{j+1}, u_{i+2}, \cdots, u_{p}\right\}$ e $B=\left\{u_{1}, u_{2}, \cdots, u_{i+1}, w_{j+2}, \cdots, w_{q}\right\}$. Essa partição consiste em transferir $i+1$ vértices de $V_{1}$ para a segunda parte da partição, bem como transferir $j+1$ vértices de $V_{2}$ para a primeira parte, o que dará origem a um corte de arestas com cardinalidade $(i+1)(j+1)+i j$, já que a inversão de posições elimina as $(i+1) j+i(j+1)$ arestas do corte original $V=\left(V_{1}, V_{2}\right)$. A Figura 4 a seguir ilustra essa situação, exibindo as partições Half Cut para os grafos $K_{5,5}$ e $K_{5,7}$.

\section{Trabalhos Futuros}

[Acharya and Gill 1981] provaram que as grades planares completas $P_{r} \times P_{s}$ são grafos graciosos. Consequentemente tais grafos admitem uma partição Half Cut. Pretendemos em artigos futuros exibir partições Half Cut para as grades planares completas $P_{r} \times P_{s}$. Já foi verificado em [Sucupira et al. 2017] que os grafos graciosos formam uma subclasse própria dos grafos Half Cut, pois há grafos do tipo Half Cut que não são graciosos, como por exemplo os grafos completos $K_{n}$ com $n=q^{2}>4$. Desejamos também estudar em que condições um grafo bipartido admite uma partição Half Cut, já que nem todo grafo bipartido é Half Cut. Um exemplo de grafo bipartido que não é Half Cut são os ciclos $C_{n} \operatorname{com} n \equiv 2(\bmod 4)$. Verificamos também em [Sucupira et al. 2017] que toda árvore é do tipo Half Cut, embora ainda continue em aberto a Conjectura de RingelKotzig [Huang et al. 1982] a qual afirma que todas as árvores são graciosas. Essa dificudade em provar a conjectura para as árvores já nos dá indícios de que não é uma tarefa 


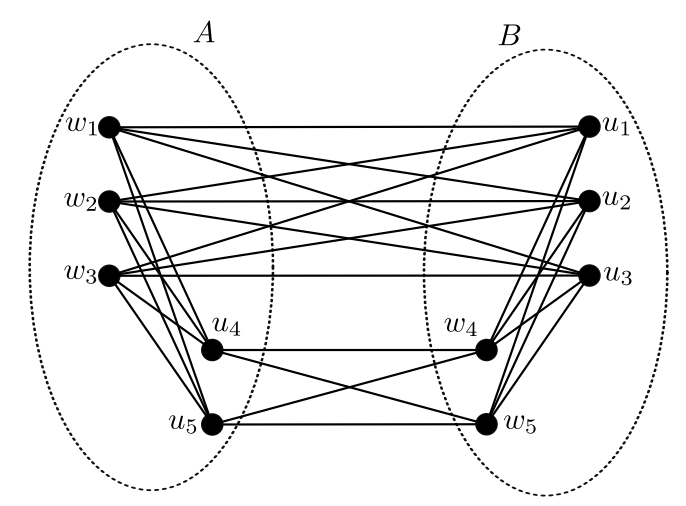

(a)

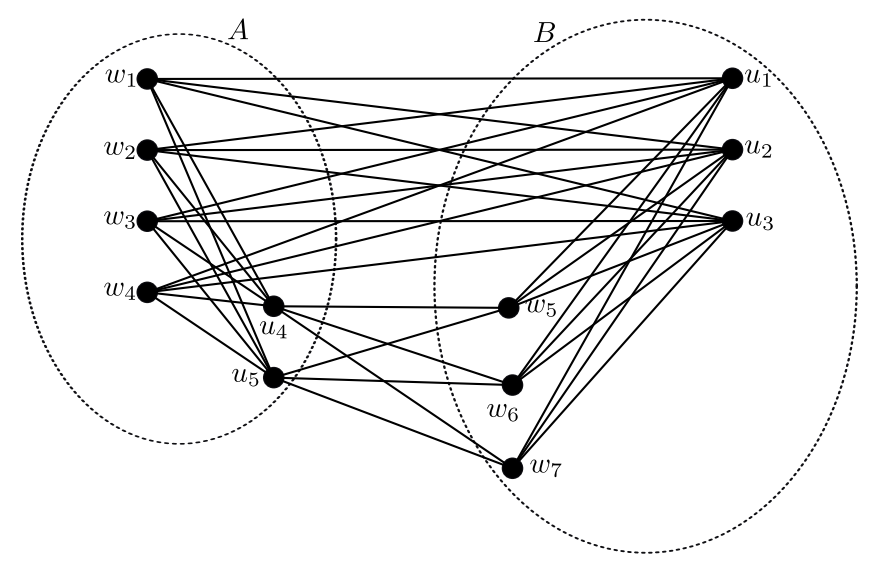

(b)

Figura 4. (a) Half cut de $K_{5,5}$ e (b) Half cut de $K_{5,7}$.

simples determinar sob que condições um grafo bipartido é gracioso. Desejamos também observar se no caso dos grafos graciosos há alguma relação entre as partições Half Cut que obtivemos e possíveis rotulações graciosas, de modo a utilizar uma partição Half Cut para determinar uma rotulação graciosa e vice-versa.

\section{Referências}

Acharya, B. and Gill, M. (1981). On the index of gracefulness of a graph and the gracefulness of two-dimensional square lattice graphs. Indian J. Math, 23:81-94.

Golomb, S. W. (1972). How to number a graph. Graph theory and computing, pages 23-37.

Huang, C., Kotzig, A., and Rosa, A. (1982). Further results on tree labellings. Util. math., $21 c$, pages $31-48$.

Rosa, A. (1966). On certain valuations of the vertices of a graph. In Theory of Graphs (Internat. Symposium, Rome, pages 349-355.

Sucupira, R., Faria, L., and Klein, S. (2017). Grafos half cut. In de Computação, S. B., editor, Anais do XXXVII Congresso da SBC, pages 115-118. XXXVII Congresso da Sociedade Brasileira de Computação. 